\title{
HODGE METRICS AND POSITIVITY OF DIRECT IMAGES
}

\author{
CHRISTOPHE MOUROUGANE, SHIGEHARU TAKAYAMA
}

\begin{abstract}
Building on Fujita-Griffiths method of computing metrics on Hodge bundles, we show that the direct image of an adjoint semi-ample line bundle by a projective submersion has a continuous metric with Griffiths semi-positive curvature. This shows that for every holomorphic semi-ample vector bundle $E$ on a complex manifold, and every positive integer $k$, the vector bundle $S^{k} E \otimes \operatorname{det} E$ has a continuous metric with Griffiths semi-positive curvature. If $E$ is ample on a projective manifold, the metric can be made smooth and Griffiths positive. ${ }^{1}$
\end{abstract}

\section{INTRODUCTION}

We study the positivity properties of direct images of adjoint line bundles. We get

Theorem 1.1. Let $\phi: Y \longrightarrow X$ be a projective submersion between two complex manifolds. Let $L$ be a semi-ample line bundle on $Y$. Then the vector bundle $\phi_{\star}\left(K_{Y / X} \otimes L\right)$ has a continuous metric with Griffiths semi-positive curvature.

Theorem 1.2. Let $\phi: Y \longrightarrow X$ be a submersion between two complex projective manifolds. Let $L$ be an ample line bundle on $Y$. Then the vector bundle $\phi_{\star}\left(K_{Y / X} \otimes L\right)$ has a smooth metric with Griffiths positive curvature.

On grassmanian manifolds, abelian varieties and toric varieties, these results were already proved either by the Castelnuovo-Mumford criterion for global generation or by trying to mimic the Frobenius morphisms over $\mathbb{C}([15],[16$, Théorème 3]). These theorems should be compared with by now classical results due to Fujita, Kawamata and others in algebraic geometry setting (see for example [14, Chapter 6.3.E]). These constructions may help to find some topological properties of algebraic sub-varieties under ampleness assumptions for the normal bundle (see for example [7] and [14] part two). The interesting outcome is that metrics could be used to construct Morse functions, whose indices are computed by the curvature.

For the main application, we consider a holomorphic vector bundle $E$ on a compact complex manifold, and intend to construct metrics on vector bundles associated to $E$ which would reflect algebraic positivity properties of $E$. Just note that on the variety $\pi: \mathbb{P}(E) \longrightarrow X$ of rank one quotients of $E$ the direct image $\pi_{\star}\left(K_{\mathbb{P}(E) / X} \otimes \mathcal{O}_{E}(k+r)\right)$, where $r$ is the rank of $E$, is $S^{k} E \otimes \operatorname{det} E$ to infer

Corollary 1.3. Let $E$ be a semi-ample vector bundle on a complex manifold. Then for all positive integer $k$, the vector bundles $S^{k} E \otimes \operatorname{det} E$ have continuous metrics with Griffiths semi-positive curvature.

Corollary 1.4. Let $E$ be an ample vector bundle on a complex projective manifold. Then for all positive integer $k$, the vector bundles $S^{k} E \otimes \operatorname{det} E$ are Griffiths positive.

The theory of resolution of the $\bar{\partial}$-equation with $L^{2}$-estimates for example shows that Griffiths positivity and ampleness are equivalent for line bundles. This implies through the curvature computation of $\mathcal{O}_{E}(1)$ that Griffiths positive vector bundles are ample. The converse is a problem raised by Griffiths [8, problem (0.9)], and solved positively on curves by Umemura [20] using the concept of stability (see also [3]). Our results provide a weak answer to Griffiths's problem. The appearance of the determinant line bundle has the same origin than its appearance in the vanishing theorem of Griffiths for the cohomology of ample vector bundles.

The first idea in the proof is to use natural cyclic coverings to be able to use Hodge metrics on the direct image of the relative canonical sheaf under a proper Kähler surjective map between two complex manifolds. This may be seen as a metric aspect of Ramanujam's idea to reduce vanishing theorem to topological properties [18] (see also [13]).

\footnotetext{
${ }^{1} 2000$ Mathematics Subject Classification : 32L05,(14F05,32J27)
} 
Applying Griffiths method of computing metrics on Hodge bundles, we will be able to compute the curvature of direct image of the structure sheaf for proper Kähler submersions. It turns out that the metric on the top direct image of the structure sheaf of the source manifold is semi-negatively curved.

We then have to determine the singularities of the Hodge metrics. For a proper surjective map $f: Z^{n+r} \longrightarrow$ $X^{n}$, the Jacobian ideal $J a c_{f}$ is the ideal of $\mathcal{O}_{Z}$ generated by the $n \times n$ - minors of the matrix of the differential of $f$ (computed in any chart). The discriminant locus $\Delta_{f} \subset X$ is defined to be the image under $f$ of the sub-scheme of $Z$ where the map $f$ is not submersive, that is the sub-scheme cut out by $J a c_{f}$. Denote $X-\Delta_{f}$ by $X^{0}$ and $f^{-1}\left(X^{0}\right)$ by $Z^{0}$. The map $f^{0}:=f_{\mid Z^{0}}: Z^{0} \longrightarrow X^{0}$ is then a projective submersion. With these notations, our main technical lemma generalizes Fujita's observation [6] lemma 1.12].

Lemma 1.5. Let $f: Z \longrightarrow X$ be a proper Kähler surjective map between two complex manifolds.

(1) Then, the Hodge metric on $\left(f^{0}\right)_{\star}\left(K_{Z^{0} / X^{0}}\right)$ extends as a metric with poles on $f_{\star}\left(K_{Z / X}\right)$.

(2) The Hodge metric on $\left(f^{0}\right)_{\star}\left(K_{Z^{0} / X^{0}}\right)$ extends as a smooth metric on $f_{\star}\left(\operatorname{Jac}_{f} \otimes K_{Z / X}\right)$.

This is far simpler than general results obtained by Kawamata, Zücker, Kollár and Cattani-Kaplan-Schimd.

This paper is a revised and expanded version of our preprint [17. Shortly before we ended the writing of our text, Bo Berndtsson gave a nice proof of the Nakano positivity of the direct image of adjoint ample line bundle in the same setting than ours [2]. We nevertheless feel worth to display our techniques for these are different from his.

Acknowledgment. The first named author warmly thanks Indranil Biswas for a collaboration in an attempt to prove similar results.

\section{THe TOOLS}

2.1. Ampleness and positivity. We refer to [11] or [14 $\S 6]$ for basics about ample vector bundles and to [8] or [4, chapter VII] for basics about positive vector bundles. All vector bundles are assumed to be holomorphic.

A vector bundle $E$ on a compact complex manifold $X$ is said to be semi-ample if for some positive integer $k$, its symmetric power $S^{k} E$ is generated by its global sections. Associated to $E$, we have $\pi: \mathbb{P}(E) \longrightarrow X$ the variety of rank one quotients of $E$ together with its tautological quotient line bundle $\mathcal{O}_{E}(1)$. The semi-ampleness of $E$ is rephrased that for every $x \in X$, every section $s_{x}$ of $\mathcal{O}_{E}(k)$ over the fiber $\mathbb{P}\left(E_{x}\right)$ extends to a global section of $\mathcal{O}_{E}(k)$ over $\mathbb{P}(E)$. This in particular implies that $\mathcal{O}_{E}(k)$ is generated by its global sections. A vector bundle $E$ is said to be ample if its associated line bundle $\mathcal{O}_{E}(1)$ is ample on $\mathbb{P}(E)$. This in particular implies the existence of an integer $k$ such that for every $x \in X$, every section $s_{x}$ of $\mathcal{O}_{E}(k)$ over the first infinitesimal neighborhood of the fiber $\mathbb{P}\left(E_{x}\right)$ extends to a global section of $\mathcal{O}_{E}(k)$ over $\mathbb{P}(E)$.

Recall the formula for the curvature of the Chern connection of the quotient metric $h_{q}$ on $\mathcal{O}_{E}(1)$ of a chosen metric $h$ on $E$. Here $a^{\star}$ parametrizes rank one quotients of $E$ and $F S\left(h_{x}\right)$ is the Fubini-Study metric of the hermitian form $h_{x}$ on $E_{x}$.

$$
\begin{aligned}
\Theta\left(\mathcal{O}_{E}(1), h_{q}\right) & =\sqrt{-1} \partial \bar{\partial} \log \left\|e_{0}^{\star}+z_{1} e_{1}^{\star}+\cdots z_{r-1} e_{r-1}^{\star}\right\|^{2} \\
& =\Theta\left(\mathcal{O}_{\mathbb{P}\left(E_{x}\right)}(1), F S\left(h_{x}\right)\right)-\frac{\left\langle\pi^{\star} \Theta\left(E^{\star}, h^{\star}\right) a^{\star}, a^{\star}\right\rangle}{\left\langle a^{\star}, a^{\star}\right\rangle} .
\end{aligned}
$$

A vector bundle $E$ is said to be Griffiths positive, if it can be endowed with a smooth hermitian metric $h$ such that for all $x \in X$ and all non-zero decomposable tensors $v \otimes e \in T X_{x} \otimes E_{x}$, the curvature term $\langle\Theta(E, h)(v, \bar{v}) e, e\rangle_{h}$ is positive, where $\Theta(E, h) \in \mathcal{C}_{1,1}^{\infty}(X, \operatorname{Herm}(E))$ is the curvature of the Chern connection $\nabla_{E, h}$ of $(E, h)$.

A continuous hermitian metric $h$ on a vector bundle $b: E \longrightarrow X$ is said to be Griffiths positive, if there exists a smooth positive real $(1,1)$-form $\omega_{X}$ on $X$ such that in the sense of currents

$$
-\sqrt{-1} \partial \bar{\partial} \log h(\xi) \geq b^{\star} \omega_{X},
$$

where $h$ is seen as a continuous quadratic function on the total space $E-X \times\{0\}$. At the points where the metric $h$ is smooth, these two notions of Griffiths positivity coincide. 
2.2. Cyclic covers. A reference for this part is [5] $\S 3]$. Take a semi-ample line bundle $L$ on a complex manifold $Y$ and fix a positive integer $k$ such that the tensor power $L^{k}$ is generated by its global sections. Then Bertini's theorem (see for example [10, page 137]) insures that a generic section $s$ of $L^{k}$ over $Y$ is transverse to the zero section (i.e. $d s_{\mid D_{s}}: T Y_{\mid D_{s}} \longrightarrow L_{\mid D_{s}}^{k}$ is surjective), and defines a smooth divisor $D_{s}:=(s=0)$. Let

$$
p=p_{s}: Y_{s} \longrightarrow Y
$$

be the cyclic covering of $Y$ obtained by taking the $k$-th root out of $D_{s}$. The space

$$
Y_{s}:=\left\{l \in L / l^{k}=s(p(l))\right\}
$$

is a smooth hypersurface of the total space $L$, and the map $p$ is a finite cover of degree $k$ totally ramified along the zero locus $D_{s}$ of $s$. The space $Y_{s}$ may also be described as the spectrum $\operatorname{Spec} \mathcal{A}_{s}$ of the algebra

$$
\mathcal{A}_{s}:=\frac{\oplus_{i=0}^{+\infty} L^{-i}}{\left(l^{\star}-\check{s}\left(l^{\star}\right), l^{\star} \in L^{-k}\right)}
$$

where $\check{s}$ is the sheaf inclusion $L^{-k} \stackrel{\times s}{\rightarrow} \mathcal{O}_{Y}$. The direct image of the structure sheaf $\mathcal{O}_{Y_{s}}$ is hence $p_{\star} \mathcal{O}_{Y_{s}}=\mathcal{A}_{s} \simeq$ $\oplus_{i=0}^{k-1} L^{-i}$.

In our setting, we consider a projective submersion $\phi: Y \longrightarrow X$ of relative dimension $r$ between two complex manifolds and $L$ a semi-ample line bundle on $Y$. A generic section of $L^{k}$ gives rise to a covering of $Y$ which we in fact regard as a family of coverings of the fibers of $\phi$. Because $p_{s}$ is a finite morphism, the spectral sequence of composition of direct image functors reduces to the following:

$$
\begin{aligned}
\mathcal{R}^{r}\left(\phi \circ p_{s}\right)_{\star} \mathcal{O}_{Y_{s}} & =\mathcal{R}^{r} \phi_{\star}\left(\mathcal{R}^{0} p_{\star} \mathcal{O}_{Y_{s}}\right) \\
& =\mathcal{R}^{r} \phi_{\star}\left(\oplus_{i=0}^{k-1} L^{-i}\right)=\oplus_{i=0}^{k-1} \phi_{\star}\left(K_{Y / X} \otimes L^{i}\right)^{\star} .
\end{aligned}
$$

Here we have used Serre duality on the fibers of the smooth morphism $\phi$.

2.3. The Hodge metric. We recall the basics on geometric variations of Hodge structures and Griffiths's computations (9, theorem 6.2]) of the curvature the Hodge metric (see also [19 § 7] and 21, chapter 10]). Here we assume that $f: Y \longrightarrow B$ is a proper Kähler submersion of relative dimension $r$, in particular we regard $f: Y \longrightarrow B$ as a smooth family of compact Kähler manifolds of dimension $r$. Fix a non-negative integer $d$. The local system $\mathcal{R}^{d} f_{\star} \mathbb{C}$ can be realized as the sheaf of germs of the flat sections of the holomorphic vector bundle $\mathbf{H}_{\mathbb{C}}^{d}$ associated with the locally free sheaf $\left(\mathcal{R}^{d} f_{\star} \mathbb{C}\right) \otimes \mathcal{O}_{B}$ endowed with the flat holomorphic connection $\nabla$ : $\mathbf{H}_{\mathbb{C}}^{d} \longrightarrow \Omega_{B}^{1} \otimes \mathbf{H}_{\mathbb{C}}^{d}$, the Gauss-Manin connection. By semi-continuity and Hodge decomposition, the vector spaces $H^{p, d-p}\left(Y_{b}, \mathbb{C}\right)(b \in B)$ have constant dimension. By elliptic theory they hence form a differentiable sub-bundle $\mathbf{H}^{p, d-p}$ of $\mathbf{H}_{\mathbb{C}}^{d}$. Denote by $\mathbf{F}^{p}$ the differentiable sub-bundle $\oplus_{i \geq p} \mathbf{H}^{i, d-i}$ of $\mathbf{H}_{\mathbb{C}}^{d}$. By a theorem of Griffiths, the $\mathbf{F}^{p}$ have natural structure of holomorphic sub-vector bundles of $\mathbf{H}_{\mathbb{C}}^{d}$. A relative Dolbeault theorem identifies $\mathbf{E}^{p}:=\mathbf{F}^{p} / \mathbf{F}^{p+1}$ with the holomorphic vector bundle associated with the locally free sheaf $\mathcal{R}^{d-p} f_{\star} \Omega_{Y / B}^{p}$.

We now recall the construction of the Hodge metric on the primitive part of $\mathbf{E}^{p}$. We fix a family $\eta_{b}(b \in B)$ of polarizations given by a section of $\mathcal{R}^{2} f_{\star} \mathbb{Z}$ (e.g. the family of Chern classes $c_{1}\left(L_{\mid Y_{b}}\right)$ for an ample line bundle $L$ on $Y$ ). By the hard Lefschetz theorem, the bilinear form on the fibers of $\mathbf{H}_{\mathbb{C}}^{d}$ given by

$$
S\left(c_{1}, c_{2}\right):=(-1)^{\frac{d(d-1)}{2}} \int_{Y_{b}} \eta_{b}^{r-d} \wedge c_{1} \wedge c_{2}
$$

is non-degenerate. We define the primitive cohomology to be $\mathbf{P}^{d}:=\operatorname{Ker}\left(\eta^{r-d+1}: \mathbf{H}^{d} \longrightarrow \mathbf{H}^{2 r-d+2}\right)$, which is also a differentiable sub-bundle of $\mathbf{H}^{d}$. By the Hodge-Riemann bilinear relations, the differentiable sub-bundles $\mathbf{H}^{p, d-p}$ and $\mathbf{H}^{p^{\prime}, d-p^{\prime}}$ are orthogonal unless $p+p^{\prime}=d$ and

$$
h(c):=(\sqrt{-1})^{p-q} S(c, \bar{c})
$$

defines a positive definite metric on $\mathbf{H}_{\text {prim }}^{p, d-p}:=\mathbf{H}^{p, d-p} \cap \mathbf{P}^{d}$. We set $\mathbf{F}_{\text {prim }}^{p}:=\mathbf{F}^{p} \cap \mathbf{P}^{d}$. Those bundle also have natural holomorphic structures. We also set $\mathbf{E}_{\text {prim }}^{p}:=\mathbf{F}_{\text {prim }}^{p} / \mathbf{F}_{\text {prim }}^{p+1}$. The fiber-wise isomorphism of $\left(\mathbf{E}_{\text {prim }}^{p}\right)_{b}$ with $H_{\text {prim }}^{p, d-p}\left(Y_{b}, \mathbb{C}\right) \subset H^{d}\left(Y_{b}, \mathbb{C}\right)$ enables to equip the holomorphic vector bundle $\mathbf{E}_{\text {prim }}^{p}$ with a smooth positive definite hermitian metric, called the Hodge metric.

We need some definitions in order to express the curvature of the corresponding Chern connection. First recall the transversality property of the Gauss-Manin connection $\nabla \mathbf{F}^{p} \subset \Omega_{B}^{1} \otimes \mathbf{F}^{p-1}$ which accounts for the 
Cartan-Lie formula of the derivative of a family of cohomology classes (see [21, proposition 9.14]). Denote by $\bar{\nabla}^{p}: \mathbf{E}^{p} \longrightarrow \Omega_{B}^{1} \otimes \mathbf{E}^{p-1}$ the $\mathcal{O}_{B}$-linear map built by first lifting to $\mathbf{F}^{p}$ applying the Gauss-Manin connection and projecting to $\mathbf{E}^{p-1}$. The second fundamental form in $\mathcal{C}_{\infty}^{1,0}\left(B, \operatorname{Hom}\left(\mathbf{F}^{p}, \mathbf{H}_{\mathbb{C}}^{d} / \mathbf{F}^{p}\right)\right)$ of the sequence

$$
0 \longrightarrow \mathbf{F}^{p} \longrightarrow \mathbf{H}_{\mathbb{C}}^{d} \longrightarrow \mathbf{H}_{\mathbb{C}}^{d} / \mathbf{F}^{p} \longrightarrow 0
$$

with respect to the Gauss-Manin connection (or equivalently with the flat metric on $\mathbf{H}_{\mathbb{C}}^{d}$ ) actually induces $\bar{\nabla}^{p}: \mathbf{E}^{p} \longrightarrow \Omega_{B}^{1} \otimes \mathbf{E}^{p-1}$. Formulas for the curvature of quotient hermitian holomorphic vector bundles then lead to

Theorem 2.1. 9, theorem 5.2] The curvature $\Theta\left(\mathbf{E}_{\text {prim }}^{p}\right)$ of the holomorphic vector bundle $\mathbf{E}_{\text {prim }}^{p}$ endowed with its Hodge metric is given by

$$
\left\langle\Theta\left(\mathbf{E}_{\text {prim }}^{p}\right)(V, \bar{V}) \sigma, \sigma\right\rangle_{\text {Hodge }}=\left\langle\bar{\nabla}_{V}^{p} \sigma, \bar{\nabla}_{V}^{p} \sigma\right\rangle_{\text {Hodge }}-\left\langle\left(\bar{\nabla}_{V}^{p+1}\right)^{\star} \sigma,\left(\bar{\nabla}_{V}^{p+1}\right)^{\star} \sigma\right\rangle_{\text {Hodge }}
$$

where $V$ is a local vector field on $B$ and $\sigma$ a local section of $\mathbf{E}^{p}$.

We now apply this result in the case of the family of the cyclic covers $\phi \circ p_{s}: Y_{s} \longrightarrow X$ obtained by taking the $k$-th root of a section $s$ of $L^{k}$ transverse to the zero section as in $\S 2.2$ We have to restrict the study over Zariski open sets outside the discriminant locus $\Sigma_{s}$ of $\phi \circ p_{s}$. It is the set of $x \in X$ where $s_{\mid Y_{x}} \in \Gamma\left(Y_{x}, L^{k}\right)$ is not transverse to the zero section. We set $X^{0}:=X-\Sigma_{s}$ and $Y_{s}^{0}:=\left(\phi \circ p_{s}\right)^{-1}\left(X-\Sigma_{s}\right)$ so that $\phi \circ p: Y_{s}^{0} \longrightarrow X^{0}$ becomes a smooth family. Then, since $\bar{\nabla}^{0}$ vanishes, the above theorem implies the following

Corollary 2.2. The vector bundle $\mathbf{E}_{\text {prim }}^{0}=\mathbf{E}^{0}=\mathcal{R}^{r}\left(\phi \circ p_{s}\right)_{\star} \mathcal{O}_{Y_{s}^{0} / X^{0}}=\oplus_{i=0}^{k-1} \mathcal{R}^{r} \phi_{\star}\left(L^{-i}\right)$ with the Hodge metric is Griffiths (actually even Nakano) semi-negative.

2.4. Singularities of the Hodge metric. We now deal with the general case, namely $f: Y \longrightarrow B$ may not be smooth. We first recall the method of Fujita [ 6 . We therefore assume that the base $B$ is one dimensional.

The Hodge metric on the direct image of the relative canonical sheaf is described as follows. Let $b \in B$ be a point and let $(U, t)$ be a local coordinate centered at $b=\{t=0\}$. A section $\omega \in \Gamma\left(U, f_{\star} K_{Y / B}\right)$ - when regarded as a section in $\Gamma\left(U, f_{\star} \operatorname{Hom}\left(f^{\star} K_{B}, K_{Y}\right)\right)$ and applied to $f^{\star} d t$ - gives a section of $K_{Y}$ on $f^{-1}(U)$ which we denote by $\omega \cdot d t$. If $\varphi_{b} \in \Gamma\left(Y_{b}, K_{Y_{b}}\right)$ fulfills the relation $\omega \cdot d t=\varphi_{b} \wedge f^{\star} d t$ over $Y_{b}$ (which amounts to saying that in the differentiable trivialization $Y_{\mid U} \simeq Y_{b} \times U$, the section $\omega$ is sent to $\left.\varphi_{b}\right)$, then the Hodge norm at $b \in B$ of the section $\omega$ is

$$
\|\omega\|_{\text {Hodge }}^{2}=(\sqrt{-1})^{n}(-1)^{\frac{n(n-1)}{2}} \int_{Y_{b}} \varphi_{b} \wedge \overline{\varphi_{b}},
$$

here $n=\operatorname{dim} Y-1$. Fujita checked that in this setting in case $\operatorname{dim} B=1$ the Hodge metric on $f_{\star} K_{Y / B}$ is bounded from below by a positive quantity and hence that the only possible singularities of the Hodge metric on $f_{\star} K_{Y / B}$ are poles (see [6] lemma 1.12]). $m)$.

We just give the typical example which occurs for a local model of our cyclic covers (for some positive integer

$$
\begin{array}{ccccc}
Y_{s}=\left\{(t, z, l) \in \mathbb{C}^{3} / l^{k}=t+z^{m}\right\} & \stackrel{p_{s}}{\longrightarrow} & Y & \stackrel{\phi}{\longrightarrow} & X=\{t \in \mathbb{C}\} \\
(t, z, l) & \mapsto & (t, z) & \mapsto & t
\end{array}
$$

The cotangent bundle $\Omega_{Y_{s}}^{1}$ is generated by $d t, d z, d l$ subject to the relation $k l^{k-1} d l-d t-m z^{m-1} d z=0$. If $\omega \cdot d t$ is written as $\eta(z, l) d z \wedge d l$ for a holomorphic function $\eta(z, l)$, then $\varphi_{0}$ may be chosen to be $\varphi_{0}=k^{-1} l^{1-k} \eta(z, l) d z$ with a pole of order $k-1$ on the fiber over $t=0$ and no singularities elsewhere.

We can now go back to the proof of our main lemma.

Proof of lemma 1.5. Let $f: Z^{n+r} \longrightarrow X^{n}$ be a proper Kähler surjective map between two complex manifolds of relative dimension $r$. Let $z=\left(z_{1}, z_{2}, \cdots, z_{n+r}\right)$ and $x=\left(x_{1}, x_{2}, \cdots, x_{n}\right)$ be local holomorphic coordinates on $Z$ and $X$ around $z_{0}$ and $x_{0}:=f\left(z_{0}\right)$. The map $f$ is locally given by $\left(f_{i}(z)\right)_{1 \leq i \leq n}$. Let $\Psi \in f_{\star}\left(K_{Z / X}\right)$ and write

$$
\begin{aligned}
\Psi \cdot f^{\star}\left(d x_{1} \wedge d x_{2} \wedge \cdots \wedge d x_{n}\right) & =\psi d z_{1} \wedge d z_{2} \wedge \cdots \wedge d z_{n+r} \\
& =\psi \operatorname{det}\left(\frac{\partial f_{i}}{\partial z_{j}}\right)_{\substack{1 \leq i \leq n \\
1 \leq j \leq n \\
4}}^{-1} f^{\star}\left(d x_{1} \wedge d x_{2} \wedge \cdots \wedge d x_{n}\right) \wedge d z_{n+1} \wedge \cdots \wedge d z_{n+r}
\end{aligned}
$$


the Hodge norm of $\Psi$ is hence

$$
\|\Psi\|_{\text {Hodge }}^{2}=\int_{Z_{x}}|\psi|^{2}\left|\operatorname{det}\left(\frac{\partial f_{i}}{\partial z_{j}}\right)_{\substack{1 \leq i \leq n \\ 1 \leq j \leq n}}\right|^{-2}(\sqrt{-1})^{r} d z_{n+1} \wedge d \overline{z_{n+1}} \cdots \wedge d z_{n+r} \wedge d \overline{z_{n+r}} .
$$

The Hodge metric can hence only acquire poles, located furthermore over the discriminant locus of $f$. If now $\Psi$ belongs to $f_{\star}\left(J a c_{f} \otimes K_{Z / X}\right)$ then $\psi$ is a combination $\psi=\sum \psi_{J} \operatorname{det}\left(\frac{\partial f_{i}}{\partial z_{j}}\right)_{\substack{1 \leq i \leq n \\ j \in J}}$, where the sum is taken over all multi-indexes $J \subset\{1,2, \cdots, n+r\}$ of length $|J|=n$, manely $J=\left\{j_{1}, j_{2}, \ldots, j_{n}\right\}$ with $1 \leq j_{1}<j_{2}<\ldots<j_{n} \leq n+r$. It follows that

$$
\Psi \cdot f^{\star}\left(d x_{1} \wedge d x_{2} \wedge \cdots \wedge d x_{n}\right)=f^{\star}\left(d x_{1} \wedge d x_{2} \wedge \cdots \wedge d x_{n}\right) \wedge\left(\sum \psi_{J} d z_{m_{1}} \wedge \cdots \wedge d z_{m_{r}}\right) .
$$

Here the last sum is taken over all multi-indexes $J \subset\{1,2, \cdots, n+r\}$ of $|J|=n$, and $\left\{m_{1}, \ldots, m_{r}\right\}=$ $\{1,2, \cdots, n+r\} \backslash J$.

In our setting, we take a global section $s$ of $L^{k}$ transverse to the zero section and consider the corresponding cyclic cover $Y_{s}$ of $Y$. Note that if the section $s$ is locally given by $\sigma\left(x_{1}, x_{2}, \cdots, x_{n}, y_{1}, y_{2}, \cdots, y_{r}\right)$ and the map $\phi$ by $\left(x_{1}, x_{2}, \cdots, x_{n}, y_{1}, y_{2}, \cdots, y_{r}\right) \mapsto\left(x_{1}, x_{2}, \cdots, x_{n}\right)$, then there is an $j$ such that $\frac{\partial s}{\partial y_{j}}\left(p_{0}\right) \neq 0$ and the functions $\left(x_{1}, x_{2}, \cdots, x_{n}, y_{1}, y_{2}, \cdots, y_{j-1}, t, y_{j+1}, \cdots, y_{r}\right)$ can be chosen as coordinates on $Y_{s}$ near $y_{0} \in$ $p_{s}^{-1}\left(y_{0}\right)$, or there is an $i$ such that $\frac{\partial s}{\partial x_{i}}\left(p_{0}\right) \neq 0$ and the coordinates on $Y_{s}$ near $y_{0}$ can be chosen to be $\left(x_{1}, x_{2}, \cdots x_{i-1}, t, x_{i+1}, \cdots, x_{n}, y_{1}, y_{2}, \cdots, y_{r}\right)$. In the first case, $\phi \circ p_{s}$ is a submersion at $y_{0}$ and its Jacobian ideal is $\mathcal{O}_{Y_{s}}$ at $y_{0}$. In the second case the Jacobian ideal contains

$$
\frac{\partial x_{i}}{\partial t}=\frac{\partial s}{\partial t}\left(\frac{\partial s}{\partial x_{i}}\right)^{-1} \sim t^{k-1}
$$

In any case it contains $p_{s}^{\star} \mathcal{O}\left(-\frac{k-1}{k} D_{s}\right)$. This has to be compared with the formula $K_{Y_{s} / Y}=p_{s}^{\star}((k-1) L)$.

\section{Proof of Theorems}

Proof of Theorem 1.1. Let $\phi: Y \longrightarrow X$ be a projective submersion of relative dimension $r$ between two complex manifolds and $L$ be a semi-ample line bundle on $Y$. Note that the relative projectivity condition is only used to ensure the local freeness of $\phi_{\star}\left(K_{Y / X} \otimes L\right)$.

We take a positive integer $k$ so that $L^{k}$ is generated by its global sections. Take a global section $s$ of $L^{k}$ transverse to the zero section and consider the corresponding cyclic cover $Y_{s}$ of $Y$. We have seen that the Hodge metric on $\mathcal{R}^{r}\left(\phi \circ p_{s}\right)_{\star} \mathcal{O}_{Y_{s}}$ is smooth (as a Hodge metric on a smooth family) non-degenerate and semi-negatively curved outside the discriminant locus $\Sigma_{s_{\alpha}}$, is continuous on $X$, and may acquire zeros at the points $x$ over which the section $s_{\mid \mathbb{P}\left(E_{x}\right)}$ is identically zero. We now explain how the semi-ampleness assumption on the line bundle $L$ helps to remove those singularities of the Hodge metric.

When $L$ is semi-ample, $D \in\left|L^{k}\right|$ moves. We choose $n+r+1$ generic sections $s_{\alpha}$ that generate $L^{k}$. The metric $h=\sum_{\alpha=1}^{n+r+1} h_{\alpha}$ on $\mathcal{R}^{r} \phi_{\star}\left(L^{-1}\right) \subset \mathcal{R}^{r}\left(\phi \circ p_{s}\right)_{\star} \mathcal{O}_{Y_{s}}$ is continuous on $X$, smooth outside $\Sigma_{h}:=\cup_{\alpha} \Sigma_{s_{\alpha}}$ and without zeros.

Next let us discuss its curvature property. Recall the formula

$$
\begin{aligned}
\frac{\langle\Theta(E, h) \xi, \xi\rangle}{\|\xi\|^{2}} & =-\sqrt{-1} \partial \bar{\partial} \log \|\xi\|^{2}+\frac{\left\langle\nabla_{E, h} \xi, \nabla_{E, h} \xi\right\rangle}{\|\xi\|^{2}}-\frac{\sqrt{-1} \partial\|\xi\|^{2} \wedge \bar{\partial}\|\xi\|^{2}}{\|\xi\|^{4}} \\
& \geq-\sqrt{-1} \partial \bar{\partial} \log \|\xi\|^{2}
\end{aligned}
$$

for a nowhere zero local holomorphic section $\xi$ of a holomorphic vector bundle $E$ equipped with a smooth hermitian metric $h$. The last two terms give the norm at $x$ of the fundamental form of the inclusion $\mathcal{O}_{X} \xi \subset E$. Take a point $x_{0}$ in $X-\Sigma_{h}$, a non-zero vector $\xi_{0} \in \mathcal{R}^{r} \phi_{\star}\left(L^{-1}\right)_{x_{0}}^{\star}$, and a nowhere zero local holomorphic section $\xi \in \Gamma\left(U, \mathcal{R}^{r} \phi_{\star}\left(L^{-1}\right)\right)$ achieving the value $\xi_{0}$ at $x_{0}$ and normal at $x_{0}$ for the metric $h$ (i.e. $\left.\nabla_{h} \xi\left(x_{0}\right)=0\right)$. Then, the last two terms in the formula (3.1) vanish at $x_{0}$. The corollary 2.2 now asserts the function log $\|\xi\|_{h_{\alpha}}^{2}-$ 
whose complex Hessian (or Levi form) is the opposite of the curvature of a line sub-bundle of $\mathcal{R}^{r} \phi_{\star}\left(L^{-1}\right)$ - is plurisubharmonic on $U$. It then follows that $\log \|\xi\|_{h}^{2}=\log \left(\sum_{\alpha}\|\xi\|_{h_{\alpha}}^{2}\right)$ is plurisubharmonic also on $U$. This gives the Griffiths semi-negativity of $\mathcal{R}^{r} \phi_{\star}\left(L^{-1}\right)$ on $X-\Sigma_{h}$. Since $h$ is continuous and $\Sigma_{h}$ is an analytic subset (of zero Lebesgue measure), we can conclude that the continuous metric $h$ on $\mathcal{R}^{r} \phi_{\star}\left(L^{-1}\right)$ is Griffiths semi-negative on the whole of $X$.

Proof of Theorem 1.2] We now assume that $L$ is ample. Here, the ampleness assumption ensures the vanishing of $\mathcal{R}^{1} \phi_{\star}\left(K_{Y / X} \otimes L\right)$ which in turn easily ensures the local freeness of $\phi_{\star}\left(K_{Y / X} \otimes L\right)$.

Let us recall Legendre-type formula applied for a metric $h=\sum_{\alpha=1}^{\ell} h_{\alpha}$ on $\mathcal{R}^{r} \phi_{\star}\left(L^{-1}\right)$ gotten from different cyclic coverings $Y_{s_{\alpha}}\left(\right.$ for a $(1,0)$-form $u,|u|^{2}$ denotes $\left.\sqrt{-1} u \wedge \bar{u}\right)$ :

$$
\sqrt{-1} \partial \bar{\partial} \log \left(\sum_{\alpha}\|\xi\|_{h_{\alpha}}^{2}\right)=\frac{\sum_{\alpha}\|\xi\|_{h_{\alpha}}^{2} \sqrt{-1} \partial \bar{\partial} \log \|\xi\|_{h_{\alpha}}^{2}}{\sum_{\alpha}\|\xi\|_{h_{\alpha}}^{2}}+\frac{\sum_{\alpha<\beta}\left|\partial \log \|\xi\|_{h_{\alpha}}^{2}-\partial \log \|\xi\|_{h_{\beta}}^{2}\right|^{2}\|\xi\|_{h_{\alpha}}^{2}\|\xi\|_{h_{\beta}}^{2}}{\left(\sum_{\alpha}\|\xi\|_{h_{\alpha}}^{2}\right)^{2}}
$$

Applying Griffiths curvature formula (Corollary 2.2) for individual covering and the formula (3.1) for a line sub-bundle we infer that in the right hand side, the first term is semi-positive. We need to add further Hodge metrics $h_{\alpha}$ to make the second term - hence the left hand side - strictly positive.

The explicit expression in formula (2.1) and (2.2) will help to translate the algebraic ampleness assumption on $L$ into a negativity property for a well chosen metric on $\mathcal{R}^{r} \phi_{\star}\left(L^{-1}\right)$. Hence, we have for $\Psi \in\left(\phi \circ p_{s}\right)_{\star}\left(K_{Y_{s} / Y}\right)$

$$
\begin{aligned}
\partial \log h_{s}(\Psi) & =\partial \log \int_{\left(\phi \circ p_{s}\right)^{-1}(x)}|\psi|^{2}\left|\left(\frac{\partial s}{\partial t}\right)^{-1} \frac{\partial s}{\partial x_{i}}\right|^{2}(\sqrt{-1})^{r} d z_{n+1} \wedge d \overline{z_{n+1}} \cdots \wedge d z_{n+r} \wedge d \overline{z_{n+r}} \\
& =\partial \log \int_{Y_{x}}|\psi|^{2}|s|^{-2 k+2}\left|\frac{1}{k} \frac{\partial s}{\partial x_{i}}\right|^{2}(\sqrt{-1})^{r} d z_{n+1} \wedge d \overline{z_{n+1}} \cdots \wedge d z_{n+r} \wedge d \overline{z_{n+r}}
\end{aligned}
$$

We now take a positive integer $k$ so large that the map

$$
H^{0}\left(Y, L^{k}\right) \longrightarrow H^{0}\left(Y, L^{k} \otimes \phi^{\star}\left(\mathcal{O}_{X} / \mathcal{M}_{x}^{3}\right)\right)
$$

is surjective for every $x \in X$. By the compactness of $Y$, we can henceforth choose enough, but a finite number of sections $s_{\alpha} \in H^{0}\left(Y, L^{k}\right)$ to ensure positivity in all the directions in the Legendre formula. This gives continuous hermitian metrics on $\phi_{\star}\left(K_{Y / X} \otimes L\right)$ with Griffiths positive curvature. Using a regularization process as described in [16] these metrics may be smoothed keeping Griffiths positivity of the curvature.

Remark 3.1. Griffiths [9, proposition 2.16] showed that the operator $\bar{\nabla}^{p}: \mathbf{E}^{p} \longrightarrow \Omega_{B}^{1} \otimes \mathbf{E}^{p-1}$ can be expressed as a cup product with the Kodaira-Spencer class $\rho \in \Omega_{B, b}^{1} \otimes H^{1}\left(Y_{b}, T Y_{b}\right)$ of the family $f: Y \longrightarrow B$ coupled with a natural pairing. In our setting, this in turn can be related with the infinitesimal displacement of the hypersurfaces $D_{s, x}$ of $Y_{x}$ given by the vanishing of the section $s_{\mid Y_{x}}$, namely (see [12, chapter 5.2 (c)])

$$
\begin{array}{ccccc}
T X & \longrightarrow & H^{0}\left(D_{s, x}, \mathcal{O}\left(D_{s, x}\right)_{\mid D_{s, x}}\right) & \left(\stackrel{\delta^{\star}}{\longrightarrow}\right. & H^{1}\left(D_{s, x}, T D_{s, x}\right) \\
v & \mapsto & \left(\partial_{\tilde{v}} s\right)_{\mid D_{s, x}} & \left(\mapsto \begin{array}{ll}
\mapsto & \rho(v)
\end{array}\right)
\end{array}
$$

where $\widetilde{v}$ is a holomorphic vector field lifting $v$ on $Y_{x}$. The map $\delta^{\star}$ is the co-boundary map in the long exact sequence associated with the short exact sequence for the normal bundle of the divisor $D_{s, x}$

$$
0 \longrightarrow T D_{s, x} \longrightarrow\left(T Y_{x}\right)_{\mid D_{s, x}} \longrightarrow \mathcal{O}\left(D_{s, x}\right)_{\mid D_{s, x}} \longrightarrow 0 \text {. }
$$

Our computations make explicit the idea that $L$ being ample, the sections $s_{\mid Y_{x}}$ move sufficiently to make the operator $\bar{\nabla}^{p}: \mathbf{E}^{p} \longrightarrow \Omega_{B}^{1} \otimes \mathbf{E}^{p-1}$ have non-zero contribution in the curvature formula.

\section{REFERENCES}

[1] Berndtsson, Bo, Curvature of vector bundles and subharmonicity of Bergman kernels, math.CV/0505470

[2] Berndtsson, Bo, Curvature of vector bundles associated to holomorphic fibrations, math.CV/0511225 a revised and expanded version of [1].

[3] Campana, F. and Flenner, H., A characterization of ample vector bundles on a curve, Math. Ann., 287, (1990), 4, 571-575.

[4] Demailly, Jean-Pierre, Complex analytic and algebraic geometry 
http://www-fourier.ujf-grenoble.fr/ demailly/books.html

[5] Esnault, Hélène and Viehweg, Eckart, Lectures on vanishing theorems, DMV Seminar 20,Birkhäuser Verlag,Basel, (1992).

[6] Fujita, Takao, On Kähler fiber spaces over curves, J. Math. Soc. Japan, 30, (1978), 4, 779-794.

[7] Fulton, William, On the topology of algebraic varieties, Algebraic geometry, Bowdoin, 1985 (Brunswick, Maine, 1985 ), Proc. Sympos. Pure Math., 46, 15-46, Amer. Math. Soc., Providence, RI, (1987).

[8] Griffiths, Phillip A., Hermitian differential geometry, Chern classes, and positive vector bundles. Global Analysis, Papers in Honor of K. Kodaira (Ed. D. C. Spencer and S. Iyanaga), 185-251. Princeton Univ. Press, 1969.

[9] Griffiths, Phillip A., Periods of integrals on algebraic manifolds. III. Some global differential-geometric properties of the period mapping, Inst. Hautes Études Sci. Publ. Math., 38, (1970), 125-180.

[10] Griffiths, Phillip and Harris, Joseph, Principles of algebraic geometry, Wiley Classics Library, Reprint of the 1978 original, John Wiley \& Sons Inc., New York, (1994).

[11] Hartshorne, Robin, Ample vector bundles, Inst. Hautes Études Sci. Publ. Math., 29, (1966), 63-94.

[12] Kodaira, Kunihiko, Complex manifolds and deformation of complex structures, Grundlehren der Mathematischen Wissenschaften, [Fundamental Principles of Mathematical Sciences] 283, Springer-Verlag, New York, (1986).

[13] Kollár, János, Higher direct images of dualizing sheaves. I, Ann. of Math. (2), 123, (1986), 1, 11-42.

Higher direct images of dualizing sheaves. II, Ann. of Math. (2), 124, (1986), 1, 171-202.

[14] Lazarsfeld, Robert, Positivity in algebraic geometry. II, Ergebnisse der Mathematik und ihrer Grenzgebiete. 3. Folge. A Series of Modern Surveys in Mathematics , 49, Springer-Verlag, Berlin, 2004.

[15] Manivel, Laurent, Vanishing theorems for ample vector bundles, Invent. Math., 127, (1997), 2, 401-416.

[16] Mourougane, Christophe, Images directes de fibrés en droites adjoints, Publ. Res. Inst. Math. Sci., Kyoto University., 33, (1997), 6, 893-916.

[17] Mourougane, Christophe and Takayama, Shigeharu, A positivity property of ample vector bundles, math.AG/0505324

[18] Ramanujam, C. P., Remarks on the Kodaira vanishing theorem, J. Indian Math. Soc. (N.S.), 36, 1972, $41-51$.

[19] Schmid, Wilfried, Variation of Hodge structure: the singularities of the period mapping, Invent. Math., 22, (1973), 211-319.

[20] Umemura, Hiroshi, Some results in the theory of vector bundles, Nagoya Math. J., 52, (1973), 97-128.

[21] Voisin, Claire, Hodge theory and complex algebraic geometry, Cambridge Studies in Advanced Mathematics, 77, Cambridge University Press, (2003).

Christophe Mourougane / Institut de Mathématiques de Jussieu / Plateau 7D / 175, rue du Chevaleret / 75013 Paris / France.

email : mourouga@math.jussieu.fr

Shigeharu Takayama / Graduate School of Mathematical Sciences / University of Tokyo / 3-8-1 Komaba / 153-8914 / Japan.

email : taka@ms.u-tokyo.ac.jp 